\title{
Double Lacunary Density and Some Inclusion Results in Locally Solid Riesz Spaces
}

\author{
S. A. Mohiuddine, ${ }^{1}$ Bipan Hazarika, ${ }^{2}$ and Abdullah Alotaibi ${ }^{1}$ \\ ${ }^{1}$ Department of Mathematics, Faculty of Science, King Abdulaziz University, P.O. Box 80203, Jeddah 21589, Saudi Arabia \\ ${ }^{2}$ Department of Mathematics, Rajiv Gandhi University, Rono Hills, Doimukh, Arunachal Pradesh 791 112, India
}

Correspondence should be addressed to S. A. Mohiuddine; mohiuddine@gmail.com

Received 21 February 2013; Accepted 13 June 2013

Academic Editor: Alberto Parmeggiani

Copyright (c) 2013 S. A. Mohiuddine et al. This is an open access article distributed under the Creative Commons Attribution License, which permits unrestricted use, distribution, and reproduction in any medium, provided the original work is properly cited.

We define the notions of double statistically convergent and double lacunary statistically convergent sequences in locally solid Riesz space and establish some inclusion relations between them. We also prove an extension of a decomposition theorem in this setup. Further, we introduce the concepts of double $\theta$-summable and double statistically lacunary summable in locally solid Riesz space and establish a relationship between these notions.

\section{Introduction}

Fast [1] and Steinhaus [2] independently introduced an extension of the usual concept of sequential limits which he called statistical convergence. Actually the idea of statistical convergence was formerly given under the name "almost convergence" by Zygmund in the first edition (Warsaw, 1935) of his celebrated monograph [3]. Schoenberg [4] and Šalát [5] gave some basic properties of statistical convergence. In 1985, Fridy [6] introduced the notion of statistically Cauchy sequence and proved that it is equivalent to the concept of statistical convergence. The notion of statistical convergence is a very useful functional tool for studying the convergence problems of numerical sequences/matrices through the concept of density. Later on it was further investigated by various authors in different frameworks (see [7-18]). Mursaleen and Edely [19] extended these concepts from single to double sequences by using two dimensional analogue of natural density of subsets of $\mathbb{N} \times \mathbb{N}$ and established relationship between statistical convergence and strongly Cesáro summable double sequences. Mohiuddine et al. [20] and Mursaleen and Mohiuddine [21] defined these notions for double sequences in fuzzy normed spaces and intuitionistic fuzzy normed spaces, respectively. Recently, Mohiuddine et al. [22] introduced these notions for double sequences in locally solid Riesz spaces and proved some interesting results. Fridy and Orhan [23] presented an interesting generalization of statistical convergence with the help of lacunary sequence and called it lacunary statistical convergence. Savaş and Patterson [24, 25] extended the notion of lacunary statistical convergence from single sequences to double sequences with the help of double lacunary density and proved some interesting results related to this concept. For more details related to the concept of lacunary statistical convergence for single and double sequences and applications to approximation theorems, we refer to [26-42].

On the other hand, the concept of Riesz space was introduced by Riesz [43]. Since then, with a view to utilize this concept in topology and analysis, many authors have extensively developed the theory of Riesz spaces along with their applications (e.g., $[7,22,44,45])$.

\section{Definitions and Notations}

In this section, we recall some of the basic concepts related to the notions of statistical convergence and lacunary sequence which we will use throughout the paper.

Let $E \subseteq \mathbb{N}$. Then the natural density of $E$ is denoted by $\delta(E)$ and is defined by

$$
\delta(E)=\lim _{n \rightarrow \infty} \frac{1}{n}|\{k \leq n: k \in E\}| \text { exists, }
$$


where the vertical bar denotes the cardinality of the respective set.

Definition 1 (see [14]). A sequence $x=\left(x_{k}\right)$ in a topological space $X$ is said to be statistically convergent to $\ell$ if for every neighborhood $V$ of $\ell$

$$
\delta\left(\left\{k \in \mathbb{N}: x_{k} \notin V\right\}\right)=0 .
$$

In this case, we write $S-\lim x=\ell$.

By a lacunary sequence $\theta=\left(k_{r}\right)$, where $k_{0}=0$, we will mean an increasing sequence of nonnegative integers with $h_{r}: k_{r}-k_{r-1} \rightarrow \infty$ as $r \rightarrow \infty$. The intervals determined by $\theta$ will be denoted by $I_{r}=\left(k_{r-1}, k_{r}\right]$ and the ratio $k_{r} / k_{r-1}$ will be defined by $q_{r}$ (see [46]).

Definition 2. Let $\theta$ be a lacunary sequence and let $I_{r}=\{k$ : $\left.k_{r-1}<k \leq k_{r}\right\}$. Let $K \subset \mathbb{N}$. The number $\delta_{\theta}(K)$ is called the lacunary density or $\theta$-density of $K$ if

$$
\delta_{\theta}(K)=\lim _{r} \frac{1}{h_{r}}\left|\left\{i \in I_{r}: i \in K\right\}\right| \text { exists. }
$$

The generalized lacunary mean is defined by

$$
t_{r}(x)=\frac{1}{h_{r}} \sum_{k \in I_{r}} x_{k} .
$$

Definition 3. A sequence $x=\left(x_{k}\right)$ is said to be $\theta$-summable to number $\ell$ if $t_{r}(x) \rightarrow \ell$ as $r \rightarrow \infty$. In this case we write that $\ell$ is the $\theta$-limit of $x$. If $\theta=\left(2^{r}\right)$, then $\theta$-summable reduces to $C_{1}$-summable (see [46]).

By the convergence of a double sequence we mean the convergence in the Pringsheim sense [47]. A double sequence $x=\left(x_{k, l}\right)$ has a Pringsheim $\operatorname{limit} L(\operatorname{denoted}$ by $P-\lim x=L)$ provided that given an $\varepsilon>0$ there exists an $n \in \mathbb{N}$ such that $\left|x_{k, l}-L\right|<\varepsilon$ whenever $k, l>n$. We will describe such an $x=\left(x_{k, l}\right)$ more briefly as " $P$-convergent."

Let $K \subset \mathbb{N} \times \mathbb{N}$, and let $K(m, n)$ denote the number of $(i, j)$ in $K$ such that $i \leq m$ and $j \leq n$ (see [19]). Then the lower natural density of $K$ is defined by $\underline{\delta}_{2}(K)=\lim _{\inf _{m, n \rightarrow \infty}}(|K(m, n)| / m n)$. In case that the sequence $(K(m, n) / m n)$ has a limit in Pringsheim's sense, then we say that $K$ has a double natural density and is defined by $P-\lim _{m, n \rightarrow \infty}(|K(m, n)| / m n)=\delta_{2}(K)$.

For example, let $K=\left\{\left(i^{2}, j^{2}\right): i, j \in \mathbb{N}\right\}$. Then

$$
\delta_{2}(K)=P-\lim _{m, n \rightarrow \infty} \frac{|K(m, n)|}{m n} \leq P_{-} \lim _{m, n \rightarrow \infty} \frac{\sqrt{m} \sqrt{n}}{m n}=0 ;
$$

that is, the set $K$ has double natural density zero, while the set $\{(i, 3 j): i, j \in \mathbb{N}\}$ has double natural density $1 / 3$.

The double sequence $\bar{\theta}=\theta_{r, s}=\left\{\left(k_{r}, l_{s}\right)\right\}$ is called double lacunary sequence if there exist two increasing sequences of integers such that (see [25])

$$
\begin{array}{ll}
k_{o}=0, & h_{r}=k_{r}-k_{r-1} \longrightarrow \infty \quad \text { as } r \longrightarrow \infty, \\
l_{o}=0, & \bar{h}_{s}=l_{s}-l_{s-1} \longrightarrow \infty \quad \text { as } s \longrightarrow \infty .
\end{array}
$$

Notations. $k_{r, s}=k_{r} l_{s}, h_{r, s}=h_{r} \overline{h_{s}}$, and $\theta_{r, s}$ is determined by

$$
\begin{aligned}
& I_{r, s}=\left\{(k, l): k_{r-1}<k \leq k_{r}, l_{s-1}<l \leq l_{s}\right\}, \\
& q_{r}=\frac{k_{r}}{k_{r-1}}, \quad \overline{q_{s}}=\frac{l_{s}}{l_{s-1}}, \quad q_{r, s}=q_{r} \overline{q_{s}} .
\end{aligned}
$$

Definition 4 (see [26]). Let $\bar{\theta}=\left\{\left(k_{r}, l_{s}\right)\right\}$ be a double lacunary sequence. Let $K \subseteq \mathbb{N} \times \mathbb{N}$. The number

$$
\delta_{\bar{\theta}}(K)=P-\lim _{r, s} \frac{1}{h_{r, s}}\left|\left\{(i, j) \in I_{r, s}:(i, j) \in K\right\}\right|
$$

is said to be double lacunary density, that is, $\bar{\theta}$-density of $K$, provided the limit exists.

We define the generalized double lacunary mean by

$$
t_{r, s}(x)=\frac{1}{h_{r, s}} \sum_{(k, l) \in I_{r, s}} x_{k, l}
$$

\section{Locally Solid Riesz Spaces}

Let $X$ be a real vector space and let $\leq$ be a partial order on this space. Then $X$ is said to be an ordered vector space if it satisfies the following properties:

(i) if $x, y \in X$ and $y \leq x$, then $y+z \leq x+z$ for each $z \in X$

(ii) if $x, y \in X$ and $y \leq x$, then $a y \leq a x$ for each $a \geq 0$.

If, in addition, $X$ is a lattice with respect to the partial order, then $X$ is said to be a Riesz space (or a vector lattice) (see [45]).

For an element $x$ of a Riesz space $X$, the positive part of $x$ is defined by $x^{+}=x \vee \overline{0}=\sup \{x, \overline{0}\}$, the negative part of $x$ by $x^{-}=(-x) \vee \overline{0}$, and the absolute value of $x$ by $|x|=x \vee(-x)$, where $\overline{0}$ is the zero element of $X$.

A subset $S$ of a Riesz space $X$ is said to be solid if $y \in S$ and $|y| \leq|x|$ implies $x \in S$.

A topological vector space $(X, \tau)$ is a vector space $X$ which has a topology (linear) $\tau$, such that the algebraic operations of addition and scalar multiplication in $X$ are continuous. Continuity of addition means that the function $f: X \times X \rightarrow$ $X$ defined by $f(x, y)=x+y$ is continuous on $X \times X$, and continuity of scalar multiplication means that the function $f: \mathbb{R} \times X \rightarrow X$ defined by $f(a, x)=a x$ is continuous on $\mathbb{R} \times X$.

Every linear topology $\tau$ on a vector space $X$ has a base $N$ for the neighborhoods of $\overline{0}$ satisfying the following properties.

(1) Each $Y \in N$ is a balanced set; that is, $a x \in Y$ holds for all $x \in Y$ and for every $a \in \mathbb{R}$ with $|a| \leq 1$.

(2) Each $Y \in N$ is an absorbing set; that is, for every $x \in$ $X$, there exists $a>0$ such that $a x \in Y$.

(3) For each $Y \in N$ there exists some $E \in N$ with $E+E \subseteq$ $Y$. 
A linear topology $\tau$ on a Riesz space $X$ is said to be locally solid [48] if $\tau$ has a base at zero consisting of solid sets. A locally solid Riesz space $(X, \tau)$ is a Riesz space equipped with a locally solid topology $\tau$.

Recall [49] that a topological space is first countable if each point has a countable (decreasing) local base.

The purpose of this paper is to give certain characterizations of lacunary statistically convergent double sequences in locally solid Riesz spaces and obtain extensions of a decomposition theorem and some inclusion results related to the notions statistically convergence and lacunary statistically convergence in locally solid Riesz spaces.

Throughout the paper, the symbol $N_{\text {sol }}$ will denote any base at zero consisting of solid sets and satisfying the conditions (1), (2), and (3) in a locally solid topology.

\section{Double Lacunary Statistical Convergence in Locally Solid Riesz Spaces}

Throughout the paper $X$ will denote the Hausdorff locally solid Riesz space which is first countable.

The idea of lacunary statistical convergence for single sequences in locally solid Riesz spaces has been recently studied by Mohiuddine and Alghamdi [50] as follows.

Definition 5 (see [50]). Let $(X, \tau)$ be a locally solid Riesz space. A sequence $\left(x_{k}\right)$ of points in $X$ is said to be $S_{\theta}(\tau)$ convergent to an element $x_{0}$ of $X$ if for each $\tau$-neighborhood $V$ of zero,

$$
\delta_{\theta}\left(\left\{k \in \mathbb{N}: x_{k}-x_{0} \notin V\right\}\right)=0 ;
$$

that is,

$$
\lim _{r} \frac{1}{h_{r}}\left|\left\{k \in I_{r}: x_{k}-x_{0} \notin V\right\}\right|=0 .
$$

In this case, we write $S_{\theta}(\tau)-\lim _{k \rightarrow \infty} x_{k}=x_{0}$ or $\left(x_{k}\right) \stackrel{S_{\theta}(\tau)}{\longrightarrow} x_{0}$.

Albayrak and Pehlivan [7] introduced the notion of statistical convergence in locally solid Riesz spaces. Afterward, Mohiuddine et al. [22] defined and studied the concept of statistical convergence in this setup as follows.

Definition 6 (see [22]). Let $(X, \tau)$ be a locally solid Riesz space. Then, a double sequence $x=\left(x_{j k}\right)$ in $X$ is said to be statistically $\tau$-convergent to the number $x_{0} \in X$ if for every $\tau$-neighborhood $V$ of zero,

$$
P-\lim _{m, n \rightarrow \infty} \frac{1}{m n}\left|\left\{(j, k), j \leq m, k \leq n: x_{j k}-x_{0} \notin V\right\}\right|=0 .
$$

In this case we write $S(\tau)-\lim x=\xi$ or $x_{j k} \stackrel{\mathcal{S ( \tau )}}{\longrightarrow} x_{0}$.

Now we recall the definition of lacunary statistical convergence of double sequences in the framework of locally solid Riesz spaces as follows.
Definition 7. Let $(X, \tau)$ be a locally solid Riesz space. A double sequence $\left(x_{k, l}\right)$ of points in $X$ is said to be double lacunary statistical $\tau$-convergent or $S_{\bar{\theta}}(\tau)$-convergent to an element $x_{0}$ of $X$ if for each $\tau$-neighborhood $V$ of zero,

$$
\delta_{\bar{\theta}}\left(\left\{(k, l) \in \mathbb{N} \times \mathbb{N}: x_{k, l}-x_{0} \notin V\right\}\right)=0 ;
$$

that is

$$
P-\lim _{r, s} \frac{1}{h_{r, s}}\left|\left\{(k, l) \in I_{r, s}: x_{k, l}-x_{0} \notin V\right\}\right|=0 .
$$

In this case, we write $S_{\bar{\theta}}(\tau)-\lim _{k, l \rightarrow \infty} x_{k, l}=x_{0}$ or $\left(x_{k, l}\right) \stackrel{S_{\bar{\theta}}(\tau)}{\longrightarrow}$ $x_{0}$.

Now we prove our results.

Theorem 8. Let $(X, \tau)$ be a locally solid Riesz space. If a double sequence $\left(x_{k, l}\right)$ of points in $X$ is $S_{\bar{\theta}}(\tau)$-convergent to $x_{0}$ in $X$, then there are double sequences $\left(y_{k, l}\right)$ and $\left(z_{k, l}\right)$ such that $S_{\bar{\theta}}(\tau)-\lim _{k, l \rightarrow \infty} y_{k, l}=x_{0}$ and $x_{k, l}=y_{k, l}+z_{k, l}$, for all $(k, l) \in$ $\mathbb{N} \times \mathbb{N}$ and $\delta_{\bar{\theta}}\left(\left\{(k, l) \in \mathbb{N} \times \mathbb{N}: x_{k, l} \neq y_{k, l}\right\}\right)=0$ and $\left(z_{k, l}\right)$ is a $S_{\bar{\theta}}(\tau)$-null sequence.

Proof. Let $\left\{V_{i}\right\}$ be a nested base of $\tau$-neighborhoods of zero. Take $n_{0}=0$ and choose an increasing sequence $\left(n_{i}\right)$ of positive integers such that

$$
\delta_{\bar{\theta}}\left(\left\{(k, l) \in \mathbb{N} \times \mathbb{N}: x_{k, l}-x_{0} \notin V_{i}\right\}\right)<\frac{1}{i} \quad \text { for } k, l>n_{i} .
$$

Let us define the sequences $\left(y_{k, l}\right)$ and $\left(z_{k, l}\right)$ as follows:

$$
y_{k, l}=x_{k, l}, \quad z_{k, l}=0, \quad \text { if } 0<k, l \leq n_{1}
$$

and suppose $n_{i}<n_{i+1}$, for $i \geq 1$,

$$
\begin{gathered}
y_{k, l}=x_{k, l}, \quad z_{k, l}=0, \quad \text { if } x_{k, l}-x_{0} \in V_{i}, \\
y_{k, l}=x_{0}, \quad z_{k, l}=x_{k, l}-x_{0}, \quad \text { if } x_{k, l}-x_{0} \notin V_{i} .
\end{gathered}
$$

To show that, (i) $P-\lim _{k, l \rightarrow \infty} y_{k, l}=x_{0}$ and (ii) $\left(z_{k, l}\right)$ is a $S_{\bar{\theta}}(\tau)$-null sequence.

(i) Let $V$ be an arbitrary $\tau$-neighborhood of zero. Since $X$ is first countable, we may choose a positive integer $i$ such that $V_{i} \subseteq V$. Then $y_{k, l}-x_{0}=x_{k, l}-x_{0} \in V_{i}$, for $k, l>n_{i}$

If $x_{k, l}-x_{0} \notin V_{i}$, then $y_{k, l}-x_{0}=x_{0}-x_{0}=0 \in V$. Hence $P$ - $\lim _{k, l \rightarrow \infty} y_{k, l}=x_{0}$.

(ii) It is enough to show that $\delta_{\bar{\theta}}(\{(k, l) \in \mathbb{N} \times \mathbb{N}$ : $\left.\left.z_{k, l} \neq 0\right\}\right)=0$. For any $\tau$-neighborhood $V$ of zero, we have

$$
\begin{aligned}
& \delta_{\bar{\theta}}\left(\left\{(k, l) \in \mathbb{N} \times \mathbb{N}: z_{k, l} \notin V\right\}\right) \\
& \quad \leq \delta_{\bar{\theta}}\left(\left\{(k, l) \in \mathbb{N} \times \mathbb{N}: z_{k, l} \neq 0\right\}\right) .
\end{aligned}
$$

If $n_{p}<k, l \leq n_{p+1}$, then

$$
\left\{(k, l) \in \mathbb{N} \times \mathbb{N}: z_{k, l} \neq 0\right\} \subseteq\left\{(k, l) \in \mathbb{N} \times \mathbb{N}: x_{k, l}-x_{0} \notin V_{p}\right\} .
$$


If $p>i$ and $n_{p}<k, l \leq n_{p+1}$, then

$$
\begin{aligned}
& \delta_{\bar{\theta}}\left(\left\{(k, l) \in \mathbb{N} \times \mathbb{N}: z_{k, l} \neq 0\right\}\right) \\
& \leq \delta_{\bar{\theta}}\left(\left\{(k, l) \in \mathbb{N} \times \mathbb{N}: x_{k, l}-x_{0} \notin V_{p}\right\}\right) \\
& <\frac{1}{p}<\frac{1}{i}<\varepsilon .
\end{aligned}
$$

This implies that $\delta_{\bar{\theta}}\left(\left\{(k, l) \in \mathbb{N} \times \mathbb{N}: z_{k, l} \neq 0\right\}\right)=0$. Hence $\left(z_{k, l}\right)$ is a $S_{\bar{\theta}}(\tau)$-null sequence.

Theorem 9. Let $(X, \tau)$ be a locally solid Riesz space and let $x=\left(x_{k, l}\right)$ be a double sequence of points in $X$. If there is a $S_{\bar{\theta}}(\tau)$-convergent sequence $y=\left(y_{k, l}\right)$ in $X$ such that $\delta_{\bar{\theta}}(\{(k, l) \in$ $\left.\left.\mathbb{N} \times \mathbb{N}: y_{k, l} \neq x_{k, l} \notin V\right\}\right)=0$, then $x$ is also $S_{\bar{\theta}}(\tau)$-convergent.

Proof. Suppose that $\delta_{\bar{\theta}}\left(\left\{(k, l) \in \mathbb{N} \times \mathbb{N}: y_{k, l} \neq x_{k, l} \notin\right.\right.$ $V\})=0$ and $S_{\bar{\theta}}(\tau)-\lim _{k, l} y_{k, l}=x_{0}$. Then for an arbitrary $\tau$ neighborhood $V$ of zero, we have

$$
\delta_{\bar{\theta}}\left(\left\{(k, l) \in \mathbb{N} \times \mathbb{N}: y_{k, l}-x_{0} \notin V\right\}\right)=0 .
$$

Now,

$$
\begin{aligned}
\{(k, l) & \left.\in \mathbb{N} \times \mathbb{N}: x_{k, l}-x_{0} \notin V\right\} \\
\subseteq & \left\{(k, l) \in \mathbb{N} \times \mathbb{N}: y_{k, l} \neq x_{k, l} \notin V\right\} \\
\cup & \left\{(k, l) \in \mathbb{N} \times \mathbb{N}: y_{k, l}-x_{0} \notin V\right\} \\
\Longrightarrow & \delta_{\bar{\theta}}\left(\left\{(k, l) \in \mathbb{N} \times \mathbb{N}: x_{k, l}-x_{0} \notin V\right\}\right) \\
\leq & \delta_{\bar{\theta}}\left(\left\{(k, l) \in \mathbb{N} \times \mathbb{N}: y_{k, l} \neq x_{k, l} \notin V\right\}\right) \\
& +\delta_{\bar{\theta}}\left(\left\{(k, l) \in \mathbb{N} \times \mathbb{N}: y_{k, l}-x_{0} \notin V\right\}\right) .
\end{aligned}
$$

Therefore, we have

$$
\delta_{\bar{\theta}}\left(\left\{(k, l) \in \mathbb{N} \times \mathbb{N}: x_{k, l}-x_{0} \notin V\right\}\right)=0 .
$$

This completes the proof of the theorem.

\section{Some Inclusions Relations in Locally Solid Riesz Spaces}

Here, we prove some inclusion type results. We begin with the following interesting result.

Theorem 10. Let $(X, \tau)$ be a locally solid Riesz space and let $x=\left(x_{k, l}\right)$ be a double sequence of points in $X$. For any double lacunary sequence $\bar{\theta}=\left\{\left(k_{r}, l_{s}\right)\right\}, S(\tau) \subseteq S_{\bar{\theta}}(\tau)$ if and only if $P$-liminf $\operatorname{ins}_{r, s} q_{r, s}>1$.

Proof. Suppose first that $P$-liminf ${ }_{r, s} q_{r, s}>1$, and $P$-liminf $\operatorname{ins}_{r, s} q_{r, s}=a$ (say). Write $b=(a-1) / 2$. Then there exists an integer $n_{0}, m_{0} \in \mathbb{N}$ such that $q_{r, s} \geq 1+b$ for $r \geq n_{0} ; s \geq m_{0}$. Hence for $r \geq n_{0} ; s \geq m_{0}$,

$$
\frac{h_{r, s}}{k_{r} l_{s}}=1-\frac{k_{r-1} l_{s-1}}{k_{r} l_{s}}=1-\frac{1}{q_{r, s}} \geq 1-\frac{1}{1+b}=\frac{b}{1+b} .
$$

Suppose that $S(\tau)-\lim _{k, l} x_{k, l}=x_{0}$. We prove that $S_{\bar{\theta}}(\tau)-\lim _{k, l} x_{k, l}=x_{0}$. Let $V$ be an arbitrary $\tau$-neighborhood of zero. Then for all $r \geq n_{0} ; s \geq m_{0}$, we have

$$
\begin{aligned}
& \frac{1}{k_{r, s}}\left|\left\{k \leq k_{r}, l \leq l_{s}: x_{k, l}-x_{0} \notin V\right\}\right| \\
& \geq \frac{1}{k_{r, s}}\left|\left\{(k, l) \in I_{r, s}: x_{k, l}-x_{0} \notin V\right\}\right| \\
& \quad=\frac{h_{r, s}}{k_{r, s}} \frac{1}{h_{r, s}}\left|\left\{(k, l) \in I_{r, s}: x_{k, l}-x_{0} \notin V\right\}\right| \\
& \geq \frac{b}{1+b} \frac{1}{h_{r, s}}\left|\left\{(k, l) \in I_{r, s}: x_{k, l}-x_{0} \notin V\right\}\right| .
\end{aligned}
$$

Since $\left(x_{k, l}\right) \stackrel{S(\tau)}{\longrightarrow} x_{0}$. Therefore this inequality implies that $\left(x_{k, l}\right) \stackrel{S_{\bar{\theta}}(\tau)}{\longrightarrow} x_{0}$. Hence $S(\tau) \subseteq S_{\bar{\theta}}(\tau)$.

Next, we suppose that $P$-lim inf ${ }_{r, s} q_{r, s}=1$. We can select a subsequence $\left\{\left(k_{r_{i}}, l_{s_{j}}\right)\right\}$ of the double lacunary sequence $\bar{\theta}$ such that

$$
\frac{k_{r_{i}} l_{s_{j}}}{k_{r_{i}-1} l_{s_{j}-1}}<1+\frac{1}{i j}, \quad \frac{k_{r_{i}-1} l_{s_{j}-1}}{k_{r_{i-1}} l_{s_{j-1}}}>i j,
$$

where $r_{i}>r_{i-1}+2, s_{j}>s_{j-1}+2$. Take $a(\neq 0) \in X$. Now we define a sequence $\left(x_{k, l}\right)$ by

$$
x_{k, l}= \begin{cases}a, & \text { if }(k, l) \in I_{r_{i}, s_{j}} \text { for some } i, j=1,2,3, \ldots \\ 0, & \text { otherwise. }\end{cases}
$$

Then $S(\tau)-\lim _{k, l} x_{k, l}=0$. To see this, let $V$ be an arbitrary $\tau$ neighborhood of zero. We choose $W \in N_{\text {sol }}$ such that $W \subseteq V$ and $a \notin W$. On the other hand, for each $m, n$ we can find a positive number $\left(i_{m}, j_{n}\right)$ such that $k_{r_{i_{m}}}<m \leq k_{r_{i_{m}+1}}, l_{s_{j_{n}}}<$ $n \leq l_{s_{j_{n}+1}}$. Then

$$
\begin{aligned}
& \frac{1}{m n}\left|\left\{k \leq m, l \leq n: x_{k, l} \notin V\right\}\right| \\
& \leq \frac{1}{k_{r_{i_{m}} s_{j_{n}}}}\left|\left\{k \leq m, l \leq n: x_{k, l} \notin W\right\}\right| \\
& \leq \frac{1}{k_{r_{i_{m}}} l_{s_{n}}}\left\{\left|\left\{k \leq k_{r_{i_{m}}}, l \leq l_{s_{j_{n}}}: x_{k, l} \notin W\right\}\right|\right. \\
& \left.\quad+\left|\left\{k_{r_{i_{m}}}<k \leq m, l_{s_{j_{n}}}<l \leq n: x_{k, l} \notin W\right\}\right|\right\} \\
& \leq \frac{1}{k_{r_{i_{m}}}, l_{s_{j_{n}}}}\left|\left\{k \leq k_{r_{i_{m}}}, l \leq l_{s_{j_{n}}}: x_{k, l} \notin W\right\}\right| \\
& \quad+\frac{1}{k_{r_{i_{m}}}, l_{s_{j_{n}}}}\left(k_{r_{i_{m}+1}}-k_{r_{i_{m}}}\right)\left(l_{s_{i_{n}+1}}-l_{s_{i_{n}}}\right) \\
& <\frac{1}{i_{m} j_{n}}+1+\frac{1}{i_{m} j_{n}}-1 \\
& <\frac{1}{\left(i_{m}+1\right)\left(j_{n}+1\right)}+\frac{1}{i_{m} j_{n}} \quad \text { for each } m, n .
\end{aligned}
$$


Therefore $S(\tau)-\lim _{k, l} x_{k, l}=0$. Now let us see that $\left(x_{k, l}\right) \notin$ $S_{\bar{\theta}}(\tau)$. Let $V$ be a $\tau$-neighborhood of zero such that $a \notin V$. Thus

$$
\begin{aligned}
& P-\lim _{i, j \rightarrow \infty} \frac{1}{h_{r_{i}, s_{j}}}\left|\left\{k_{r_{i}-1}<k \leq k_{r_{i}}, l_{s_{j}-1}<l \leq l_{s_{j}}: x_{k, l} \notin V\right\}\right| \\
& =P-\lim _{i, j \rightarrow \infty} \frac{1}{h_{r_{i}, s_{j}}}\left(k_{r_{i}, s_{j}}-k_{r_{i}-1, s_{j}-1}\right) \\
& =P-\lim _{i, j \rightarrow \infty} \frac{1}{h_{r_{i}, s_{j}}} h_{r_{i}, s_{j}}=1
\end{aligned}
$$

and for $r \neq r_{i}, \quad s \neq s_{j}, i, \quad j=1,2,3, \ldots$,

$$
\begin{aligned}
& P-\lim _{r, s \rightarrow \infty} \frac{1}{h_{r, s}}\left|\left\{k_{r-1}<k \leq k_{r}, l_{s-1}<l \leq l_{s}: x_{k, l}-a \notin V\right\}\right| \\
& \quad=1 .
\end{aligned}
$$

Hence neither $a$ nor 0 can be double lacunary statistical limit of $\left(x_{k, l}\right)$. No other point of $X$ can be double lacunary statistical limit of the sequence $\left(x_{k, l}\right)$ as well. Thus $\left(x_{k, l}\right) \notin S_{\bar{\theta}}(\tau)$. This completes the proof of the theorem.

Theorem 11. Let $(X, \tau)$ be a locally solid Riesz space and let $x=\left(x_{k, l}\right)$ be sequence in $X$. For any double lacunary sequence $\bar{\theta}=\left\{\left(k_{r}, l_{s}\right)\right\}, S_{\bar{\theta}}(\tau) \subseteq S(\tau)$ if and only if $P$ - lim $\sup _{r, s} q_{r, s}<$ $\infty$.

Proof. Suppose that $P$-lim $\sup _{r, s} q_{r, s}<\infty$. Then there exists an $H>0$ such that $q_{r, s}<H$ for all $r$, s. Let $S_{\bar{\theta}}(\tau)-\lim _{k, l} x_{k, l}=$ $x_{0}$. Let $V$ be an arbitrary $\tau$-neighborhood of zero. Let $\varepsilon>0$. We write

$$
M_{r, s}=\left\{(k, l) \in I_{r, s}: x_{k, l}-x_{0} \notin V\right\} .
$$

By the definition of double lacunary statistical convergence, there are positive numbers $r_{0}, s_{0}$ such that

$$
\frac{M_{r, s}}{h_{r, s}}<\frac{\varepsilon}{2 H} \quad \forall r>r_{0}, s>s_{0} .
$$

Let $M=\max \left\{M_{r, s}: 1 \leq r \leq r_{0}, 1 \leq s \leq s_{0}\right\}$ and let $m$, $n$ be two integers satisfying $k_{r-1}<m \leq k_{r}$; $l_{s-1}<n \leq l_{s}$; then we can write

$$
\begin{gathered}
\frac{1}{m n}\left|\left\{k \leq m, l \leq n: x_{k, l}-x_{0} \notin V\right\}\right| \\
\leq r_{0} s_{0} \frac{M}{k_{r-1} l_{s-1}}+\varepsilon \frac{1}{2 H} q_{r, s} .
\end{gathered}
$$

Since $P$ - $\lim _{r, s \rightarrow \infty} k_{r, s}=\infty$, there exist positive integers $r_{1} \geq$ $r_{0}, s_{1} \geq s_{0}$ such that

$$
\frac{1}{k_{r-1} l_{s-1}}<\frac{\varepsilon}{2 r_{0} s_{0} M} \quad \text { for } r>r_{1}, s>s_{1} .
$$

Hence for $r>r_{1}, s>s_{1}$

$$
\frac{1}{m n}\left|\left\{k \leq m, l \leq n: x_{k, l}-x_{0} \notin V\right\}\right|<\frac{\varepsilon}{2}+\frac{\varepsilon}{2}=\varepsilon .
$$

It follows that $S(\tau)-\lim _{k, l} x_{k, l}=x_{0}$.

Next we suppose that $P$-lim $\sup _{r, s} q_{r, s}=\infty$. Take an element $a \neq 0 \in X$. Let $\left\{\left(k_{r_{i}}, l_{s_{j}}\right)\right\}$ be a subsequence of the double lacunary sequence $\bar{\theta}=\left\{\left(k_{r}, l_{s}\right)\right\}$ such that $q_{r_{i}, s_{j}}>$ $i j, k_{r_{i}}>i+3, l_{s_{j}}>j+3$. Define a sequence $\left(x_{k, l}\right)$ by

$$
x_{k, l}=\left\{\begin{array}{rr}
a, \quad \begin{array}{rl}
\text { if } k_{r_{i}-1}<k \leq 2 k_{r_{i}-1} ; \quad l_{s_{j}-1}<l \leq 2 l_{s_{j}-1} \\
\\
\text { for some } i, j=1,2,3, \ldots
\end{array} \\
0, \quad \text { otherwise. }
\end{array}\right.
$$

Let $V$ be a $\tau$-neighborhood of zero such that $a \notin V$. Then for $i, j>1$

$$
\begin{aligned}
& \frac{1}{h_{r_{i}, s_{j}}}\left|\left\{k \leq k_{r_{i}}, l \leq l_{s_{j}}: x_{k, l} \notin V\right\}\right| \\
& \quad<\frac{k_{r_{i}, s_{j}-1}}{h_{r_{i}, s_{j}}}=\frac{k_{r_{i}, s_{j}-1}}{k_{r_{i}, s_{j}}-k_{r_{i}, s_{j}-1}}<\frac{1}{i j-1} .
\end{aligned}
$$

Hence $\left(x_{k, l}\right) \in S_{\bar{\theta}}(\tau)$. But $\left(x_{k, l}\right) \notin S(\tau)$, because

$$
\begin{aligned}
& \frac{1}{2 k_{r_{i}, s_{j}-1}}\left|\left\{k \leq 2 k_{r_{i}-1}, l \leq 2 l_{s_{j}-1}: x_{k, l} \notin V\right\}\right| \\
& =\frac{1}{2 k_{r_{i}, s_{j}-1}}\left[k_{r_{1}, s_{1}-1}+k_{r_{2}, s_{2}-1}+\cdots+k_{r_{i}, s_{j}-1}\right]>\frac{1}{2} .
\end{aligned}
$$

This completes the proof of the theorem.

Corollary 12. Let $(X, \tau)$ be a locally solid Riesz space and let $x=\left(x_{k, l}\right)$ be a double sequence in $X$. For any double lacunary sequence $\bar{\theta}=\left\{\left(k_{r}, l_{s}\right)\right\}, S_{\bar{\theta}}(\tau)=S(\tau)$ if and only if $1<P-\lim \inf _{r, s} q_{r, s} \leq P-\lim \sup _{r, s} q_{r, s}<\infty$.

Theorem 13. Let $(X, \tau)$ be a locally solid Riesz space, let $x=$ $\left(x_{k, l}\right)$ be a double sequence in $X$. For any double lacunary sequence $\bar{\theta}=\left\{\left(k_{r}, l_{s}\right)\right\}$, if $x=\left(x_{k, l}\right) \in S_{\bar{\theta}}(\tau) \cap S(\tau)$, then $S(\tau)-\lim _{k, l \rightarrow \infty} x_{k, l}=S_{\bar{\theta}}(\tau)-\lim _{k, l \rightarrow \infty} x_{k, l}$.

Proof. Let $x=\left(x_{k, l}\right) \in S_{\bar{\theta}}(\tau) \cap S(\tau)$ and $S(\tau)-\lim _{k, l \rightarrow \infty} x_{k, l}=$ $x_{0}$, and $S_{\bar{\theta}}(\tau)-\lim _{k, l \rightarrow \infty} x_{k, l}=y_{0}$. Suppose that $x_{0} \neq y_{0}$. Since $X$ is a Hausdorff, then there exists a $\tau$-neighborhood $V$ of zero value such that $x_{0}-y_{0} \notin V$. We choose $W \in N_{\text {sol }}$ such that $W+W \subseteq V$. Then, we have

$$
\begin{aligned}
\frac{1}{k_{m} l_{n}}\left|\left\{k \leq k_{m}, l \leq l_{n}: x_{0}-y_{0} \notin V\right\}\right| \\
\leq \frac{1}{k_{m} l_{n}}\left|\left\{k \leq k_{m}, l \leq l_{n}: x_{k, l}-x_{0} \notin W\right\}\right| \\
\quad+\frac{1}{k_{m} l_{n}}\left|\left\{k \leq k_{m}, l \leq l_{n}: y_{0}-x_{k, l} \notin W\right\}\right| .
\end{aligned}
$$


It follows from this inequality that

$$
\begin{aligned}
1 \leq & \frac{1}{k_{m} l_{n}}\left|\left\{k \leq k_{m}, l \leq l_{n}: x_{k, l}-x_{0} \notin W\right\}\right| \\
& +\frac{1}{k_{m} l_{n}}\left|\left\{k \leq k_{m}, l \leq l_{n}: y_{0}-x_{k, l} \notin W\right\}\right| .
\end{aligned}
$$

We write

$$
\begin{aligned}
& \frac{1}{k_{m} l_{n}}\left|\left\{k \leq k_{m}, l \leq l_{n}: y_{0}-x_{k, l} \notin W\right\}\right| \\
& =\frac{1}{k_{m}, l_{n}}\left|\left\{(k, l) \in \bigcup_{r, s=1}^{m, n} I_{r, s}: y_{0}-x_{k, l} \notin W\right\}\right| \\
& =\frac{1}{k_{m} l_{n}} \sum_{r, s=1}^{m, n}\left|\left\{(k, l) \in I_{r, s}: y_{0}-x_{k, l} \notin W\right\}\right| \\
& =\left(\sum_{r, s=1}^{m, n} h_{r, s}\right)^{-1}\left(\sum_{r, s=1}^{m, n} h_{r, s} \cdot T_{r, s}\right),
\end{aligned}
$$

where

$$
T_{r, s}=\frac{1}{h_{r, s}}\left|\left\{(k, l) \in I_{r, s}: y_{0}-x_{k, l} \notin W\right\}\right| .
$$

Since $S_{\bar{\theta}}(\tau)-\lim _{k, l \rightarrow \infty} x_{k, l}=y_{0}$, we have $P-\lim _{r, s \rightarrow \infty} T_{r, s}=0$. Therefore the regular weighted mean transform of $\left(T_{r, s}\right)$ also tends to 0 ; that is,

$$
P-\lim _{m, n \rightarrow \infty} \frac{1}{k_{m} l_{n}}\left|\left\{k \leq k_{m}, l \leq l_{n}: y_{0}-x_{k, l} \notin W\right\}\right|=0 .
$$

Also since $S(\tau)-\lim _{k, l \rightarrow \infty} x_{k, l}=x_{0}$, we have

$$
P-\lim _{m, n \rightarrow \infty} \frac{1}{k_{m} l_{n}}\left|\left\{k \leq k_{m}, l \leq l_{n}: x_{k, l}-x_{0} \notin W\right\}\right|=0 .
$$

From (39), (43), and (44), we have

$$
\frac{1}{k_{m} l_{n}}\left|\left\{k \leq k_{m}, l \leq l_{n}: x_{0}-y_{0} \notin V\right\}\right|=0 .
$$

This contradiction completes the proof of the theorem.

\section{Double Statistical Lacunary Summable in Locally Solid Riesz Spaces}

In this section, we introduce some new concepts by using the notions of statistical lacunary summable for double sequences.

Definition 14. Let $(X, \tau)$ be a locally solid Riesz space. A sequence $x=\left(x_{k, l}\right)$ is said to be double lacunary summable (or shortly, $\theta$-summable) in $(X, \tau)$ or simply $\theta_{\tau}$-summable to an element $x_{0} \in X$ if for each $\tau$-neighborhood $V$ of zero value that $t_{r, s}(x)-x_{0} \in V$, where $t_{r, s}(x)=\left(1 / h_{r, s}\right) \sum_{(k, l) \in I_{r, s}} x_{k, l}$. In this case, we write $\theta_{\tau}-\lim x=x_{0}$.
Definition 15. Let $(X, \tau)$ be a locally solid Riesz space. A sequence $\left(x_{k, l}\right)$ of points in $X$ is said to be double statistical lacunary $\tau$-summable or simply $S_{\delta_{\bar{\theta}}}(\tau)$-summable to an element $x_{0}$ of $X$ if for each $\tau$-neighborhood $V$ of zero value, the set $K(\bar{\theta})=\left\{(r, s) \in \mathbb{N} \times \mathbb{N}: t_{r, s}(x)-x_{0} \notin V\right\}$ has double natural density zero; that is, $\delta_{2}(K(\bar{\theta}))=0$

That is

$$
P-\lim _{m n} \frac{1}{m n}\left|\left\{r \leq m, s \leq n: t_{r, s}(x)-x_{0} \notin V\right\}\right|=0 .
$$

In this case, we write $S_{\delta_{\bar{\theta}}}(\tau)-\lim x_{k, l}=x_{0}$ or $\left(x_{k, l}\right) \stackrel{S_{\delta_{\bar{\theta}}}(\tau)}{\longrightarrow} x_{0}$.

Theorem 16. Let $(X, \tau)$ be a locally solid Riesz space. A double sequence $x=\left(x_{k, l}\right)$ in $X$ is $S_{\delta_{\bar{\theta}}}(\tau)$-summable to $x_{0}$ if and only if there exists a set $K=\{(r, s)\} \subseteq \mathbb{N} \times \mathbb{N}, r, s=1,2, \ldots$, such that $\delta_{2}(K)=1$ and $\theta_{\tau}-\lim _{(r, s) \in K} x_{r, s}=x_{0}$.

Proof. Let $V$ be an arbitrary $\tau$-neighborhood of zero. Suppose that $\theta_{\tau}-\lim _{(r, s) \in K} x_{r, s}=x_{0}$; there exists a set $K=\{(r, s)\} \subseteq$ $\mathbb{N} \times \mathbb{N}, r, s=1,2, \ldots$, with $\delta_{2}(K)=1$ and $N=N(V), M=$ $M(V)$ such that $\left(t_{r, s}(x)-x_{0}\right) \in V$ for $r>N$ and $s>M$. Write $K_{V}=\left\{(r, s) \in \mathbb{N} \times \mathbb{N}: t_{r, s}(x)-x_{0} \notin V\right\}$ and $K_{1}=$ $\left\{\left(r_{N+1}, s_{M+1}\right),\left(r_{N+2}, s_{M+2}\right), \ldots\right\}$. Then $\delta_{2}\left(K_{1}\right)=1$ and $K_{V} \subseteq$ $\mathbb{N} \times \mathbb{N}-K_{1}$ which implies that $\delta_{2}\left(K_{V}\right)=0$. Hence $x=\left(x_{k, l}\right)$ is $S_{\delta_{\bar{\theta}}}(\tau)$-summable to $x_{0}$.

Conversely suppose that $x=\left(x_{k, l}\right)$ is $S_{\delta_{\bar{\theta}}}(\tau)$-convergent to $x_{0}$. Fix a countable local base $V_{1} \supset V_{2} \supset \cdots$ at $x_{0}$. For each $i \in \mathbb{N}$, put

$$
K_{i}=\left\{(r, s) \in \mathbb{N} \times \mathbb{N}: t_{r, s}(x)-x_{0} \notin V_{i}\right\} .
$$

By hypothesis $\delta_{2}\left(K_{i}\right)=0$ for each $i$. Since the ideal $\mathscr{I}$ of all subsets of $\mathbb{N} \times \mathbb{N}$ having double density zero is a $P$-ideal (see e.g., [51]), then there exists a sequence of sets $\left(J_{i}\right)_{i}$ such that the symmetric difference $K_{i} \Delta J_{i}$ is a finite set for any $i \in \mathbb{N}$ and $J:=\cup_{i=1}^{\infty} J_{i} \in \mathscr{I}$.

Let $K=\mathbb{N} \times \mathbb{N} \backslash J$, then $\delta(K)=1$. In order to prove the theorem, it is enough to check that $\lim _{(r, s) \in K} t_{r, s}(x)=x_{0}$.

Let $i \in \mathbb{N}$. Since $K_{i} \Delta J_{i}$ is a finite, there is $\left(r_{i}, s_{i}\right) \in \mathbb{N} \times \mathbb{N}$, without loss of generality with $\left(r_{i}, s_{i}\right) \in K, r_{i}, s_{i}>i$, such that

$$
\begin{aligned}
& \left(\mathbb{N} \times \mathbb{N} \backslash J_{i}\right) \cap\left\{(r, s) \in \mathbb{N} \times \mathbb{N}: r \geq r_{i}, s \geq s_{i}\right\} \\
& \quad=\left(\mathbb{N} \times \mathbb{N} \backslash K_{i}\right) \cap\left\{(r, s) \in \mathbb{N} \times \mathbb{N}: r \geq r_{i}, s \geq s_{i}\right\} .
\end{aligned}
$$

If $(r, s) \in K$ and $r \geq r_{i}, s \geq s_{i}$ then $(r, s) \notin J_{i}$, and by (48) $(r, s) \notin K_{i}$. Thus $t_{r, s}(x)-x_{0} \in V_{i}$. So we have proved that for all $i \in \mathbb{N}$ there is $\left(r_{i}, s_{i}\right) \in K, r_{i}, s_{i}>i$, with $t_{r, s}(x)-x_{0} \in V_{i}$ for every $r \geq r_{i}, s \geq s_{i}$ : without loss of generality, we can suppose $r_{i+1}>r_{i}$ and $s_{i+1}>s_{i}$ for every $i \in \mathbb{N}$. The assertion follows taking into account that the $V_{i}^{\prime}$ s form a countable local base at $x_{0}$.

\section{Acknowledgment}

The authors gratefully acknowledge the financial support from King Abdulaziz University, Jeddah, Saudi Arabia. 


\section{References}

[1] H. Fast, "Sur la convergence statistique," Colloquium Mathematicum, vol. 2, pp. 241-244, 1951.

[2] H. Steinhaus, "Sur la convergence ordinate et la convergence asymptotique," Colloquium Mathematicum, vol. 2, pp. 73-84, 1951.

[3] A. Zygmund, Trigonometric Series, Cambridge University Press, Cambridge, UK, 2nd edition, 1979.

[4] I. J. Schoenberg, "The integrability of certain functions and related summability methods. ," The American Mathematical Monthly, vol. 66, pp. 361-375, 1959.

[5] T. Šalát, "On statistically convergent sequences of real numbers," Mathematica Slovaca, vol. 30, no. 2, pp. 139-150, 1980.

[6] J. A. Fridy, "On statistical convergence," Analysis, vol. 5, no. 4, pp. 301-313, 1985.

[7] H. Albayrak and S. Pehlivan, "Statistical convergence and statistical continuity on locally solid Riesz spaces," Topology and its Applications, vol. 159, no. 7, pp. 1887-1893, 2012.

[8] H. Cakalli, "On statistical convergence in topological groups," Pure and Applied Mathematika Sciences, vol. 43, no. 1-2, pp. 2731, 1996.

[9] H. Çakalli and M. K. Khan, "Summability in topological spaces," Applied Mathematics Letters, vol. 24, no. 3, pp. 348-352, 2011.

[10] H. Çakalli, "Lacunary statistical convergence in topological groups," Indian Journal of Pure and Applied Mathematics, vol. 26, no. 2, pp. 113-119, 1995.

[11] H. Çakalli and E. Savaş, "Statistical convergence of double sequences in topological groups," Journal of Computational Analysis and Applications, vol. 12, no. 2, pp. 421-426, 2010.

[12] A. Caserta, G. Di Maio, and L. D. R. Kočinac, "Statistical convergence in function spaces," Abstract and Applied Analysis, vol. 2011, Article ID 420419, 11 pages, 2011.

[13] I. J. Maddox, "Statistical convergence in a locally convex space," Mathematical Proceedings of the Cambridge Philosophical Society, vol. 104, no. 1, pp. 141-145, 1988.

[14] G. Di Maio and L. D. R. Kočinac, "Statistical convergence in topology," Topology and its Applications, vol. 156, no. 1, pp. 2845, 2008 .

[15] S. A. Mohiuddine, A. Alotaibi, and M. Mursaleen, "Statistical convergence through de la Vallée-Poussin mean in locally solid Riesz spaces," Advances in Difference Equations, vol. 2013, p. 66, 2013.

[16] S. A. Mohiuddine and Q. M. D. Lohani, "On generalized statistical convergence in intuitionistic fuzzy normed space," Chaos, Solitons \& Fractals, vol. 42, no. 3, pp. 1731-1737, 2009.

[17] S. A. Mohiuddine, H. Şevli, and M. Cancan, "Statistical convergence in fuzzy 2-normed space," Journal of Computational Analysis and Applications, vol. 12, no. 4, pp. 787-798, 2010.

[18] M. Mursaleen and S. A. Mohiuddine, "On ideal convergence in probabilistic normed spaces," Mathematica Slovaca, vol. 62, no. 1, pp. 49-62, 2012.

[19] M. Mursaleen and O. H. H. Edely, "Statistical convergence of double sequences," Journal of Mathematical Analysis and Applications, vol. 288, no. 1, pp. 223-231, 2003.

[20] S. A. Mohiuddine, H. Sevli, and M. Cancan, "Statistical convergence of double sequences in fuzzy normed spaces," Filomat, vol. 26, no. 4, pp. 673-681, 2012.

[21] M. Mursaleen and S. A. Mohiuddine, "Statistical convergence of double sequences in intuitionistic fuzzy normed spaces," Chaos, Solitons \& Fractals, vol. 41, no. 5, pp. 2414-2421, 2009.
[22] S. A. Mohiuddine, A. Alotaibi, and M. Mursaleen, "Statistical convergence of double sequences in locally solid Riesz spaces," Abstract and Applied Analysis, vol. 2012, Article ID 719729, 9 pages, 2012.

[23] J. A. Fridy and C. Orhan, "Lacunary statistical convergence," Pacific Journal of Mathematics, vol. 160, no. 1, pp. 43-51, 1993.

[24] R. F. Patterson and E. Savaş, "Lacunary statistical convergence of double sequences," Mathematical Communications, vol. 10, no. 1, pp. 55-61, 2005.

[25] E. Savaş and R. F. Patterson, "Lacunary statistical convergence of multiple sequences," Applied Mathematics Letters, vol. 19, no. 6, pp. 527-534, 2006.

[26] C. Çakan, B. Altay, and H. Çoşkun, "Double lacunary density and lacunary statistical convergence of double sequences," Studia Scientiarum Mathematicarum Hungarica, vol. 47, no. 1, pp. 35-45, 2010.

[27] J. A. Fridy and C. Orhan, "Lacunary statistical summability," Journal of Mathematical Analysis and Applications, vol. 173, no. 2, pp. 497-504, 1993.

[28] J. Li, "Lacunary statistical convergence and inclusion properties between lacunary methods," International Journal of Mathematics and Mathematical Sciences, vol. 23, no. 3, pp. 175-180, 2000.

[29] S. A. Mohiuddine and E. Savaş, "Lacunary statistically convergent double sequences in probabilistic normed spaces," Annali dell'Universitá di Ferrara, vol. 58, no. 2, pp. 331-339, 2012.

[30] S. A. Mohiuddine and M. Aiyub, "Lacunary statistical convergence in random 2-normed spaces," Applied Mathematics \& Information Sciences, vol. 6, no. 3, pp. 581-585, 2012.

[31] S. A. Mohiuddine, A. Alotaibi, and S. M. Alsulami, "Ideal convergence of double sequences in random 2-normed spaces," Advances in Difference Equations, vol. 2012, p. 149, 2012.

[32] M. Mursaleen, C. Çakan, S. A. Mohiuddine, and E. Savaş, "Generalized statistical convergence and statistical core of double sequences," Acta Mathematica Sinica, vol. 26, no. 11, pp. 2131-2144, 2010.

[33] M. Mursaleen and S. A. Mohiuddine, "On ideal convergence of double sequences in probabilistic normed spaces," Mathematical Reports, vol. 12(62), no. 4, pp. 359-371, 2010.

[34] M. Mursaleen, S. A. Mohiuddine, and O. H. H. Edely, "On the ideal convergence of double sequences in intuitionistic fuzzy normed spaces," Computers \& Mathematics with Applications, vol. 59, no. 2, pp. 603-611, 2010.

[35] M. Mursaleen and S. A. Mohiuddine, "On lacunary statistical convergence with respect to the intuitionistic fuzzy normed space," Journal of Computational and Applied Mathematics, vol. 233, no. 2, pp. 142-149, 2009.

[36] E. Savas, "On lacunary double statistical convergence in locally solid Riesz spaces," Journal of Inequalities and Applications, p. 201399, 2013.

[37] E. Savaş and S. A. Mohiuddine, " $\bar{\lambda}$-statistically convergent double sequences in probabilistic normed spaces," Mathematica Slovaca, vol. 62, no. 1, pp. 99-108, 2012.

[38] O. H. H. Edely, S. A. Mohiuddine, and A. K. Noman, "Korovkin type approximation theorems obtained through generalized statistical convergence," Applied Mathematics Letters, vol. 23, no. 11, pp. 1382-1387, 2010.

[39] S. A. Mohiuddine, A. Alotaibi, and M. Mursaleen, "Statistical summability $(C, 1)$ and a Korovkin type approximation theorem," Journal of Inequalities and Applications, vol. 2012, p. 172, 2012. 
[40] C. Belen and S. A. Mohiuddine, "Generalized weighted statistical convergence and application," Applied Mathematics and Computation, vol. 219, no. 18, pp. 9821-9826, 2013.

[41] S. A. Mohiuddine and A. Alotaibi, "Statistical convergence and approximation theorems for functions of two variables," Journal of Computational Analysis and Applications, vol. 15, no. 2, pp. 218-223, 2013.

[42] S. A. Mohiuddine and A. Alotaibi, "Korovkin second theorem via statistical summability $(C, 1)$," Journal of Inequalities and Applications, vol. 2013, p. 149, 2013.

[43] F. Riesz, "Sur la decomposition des operations fonctionelles lineaires," in Atti del Congresso Internazionale dei Matematici, vol. 3, pp. 143-148, Zanichelli, Bologna, Italy, 1930.

[44] W. A. J. Luxemburg and A. C. Zaanen, Riesz Spaces I, NorthHolland, Amsterdam, The Netherlands, 1971.

[45] A. C. Zaanen, Introduction to Operator Theory in Riesz Spaces, Springer, Berlin, Germany, 1997.

[46] A. R. Freedman, J. J. Sember, and M. Raphael, "Some Cesàrotype summability spaces," Proceedings of the London Mathematical Society, vol. 37, no. 3, pp. 508-520, 1978.

[47] A. Pringsheim, "Zur Theorie der zweifach unendlichen Zahlenfolgen," Mathematische Annalen, vol. 53, no. 3, pp. 289-321, 1900.

[48] G. T. Roberts, “Topologies in vector lattices,” vol. 48, pp. 533546, 1952.

[49] R. Engelking, General Topology, Heldermann, Berlin, Germany, 1989.

[50] S. A. Mohiuddine and M. A. Alghamdi, "Statistical summability through a lacunary sequence in locally solid Riesz spaces," Journal of Inequalities and Applications, vol. 2012, p. 225, 2012.

[51] I. Farah, "Analytic quotients: theory of liftings for quotients over analytic ideals on the integers," Memoirs of the American Mathematical Society, vol. 48, no. 702, p. 177, 2000. 


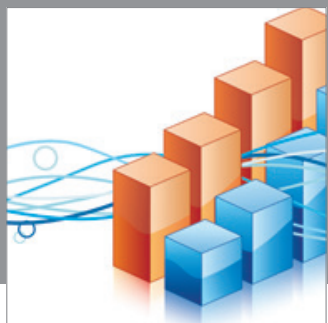

Advances in

Operations Research

mansans

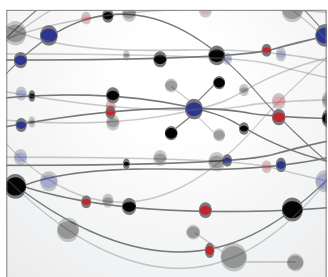

The Scientific World Journal
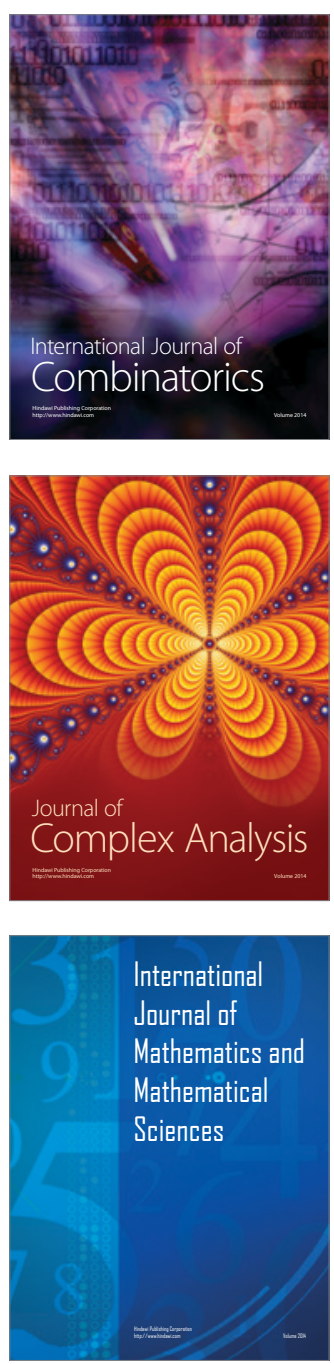
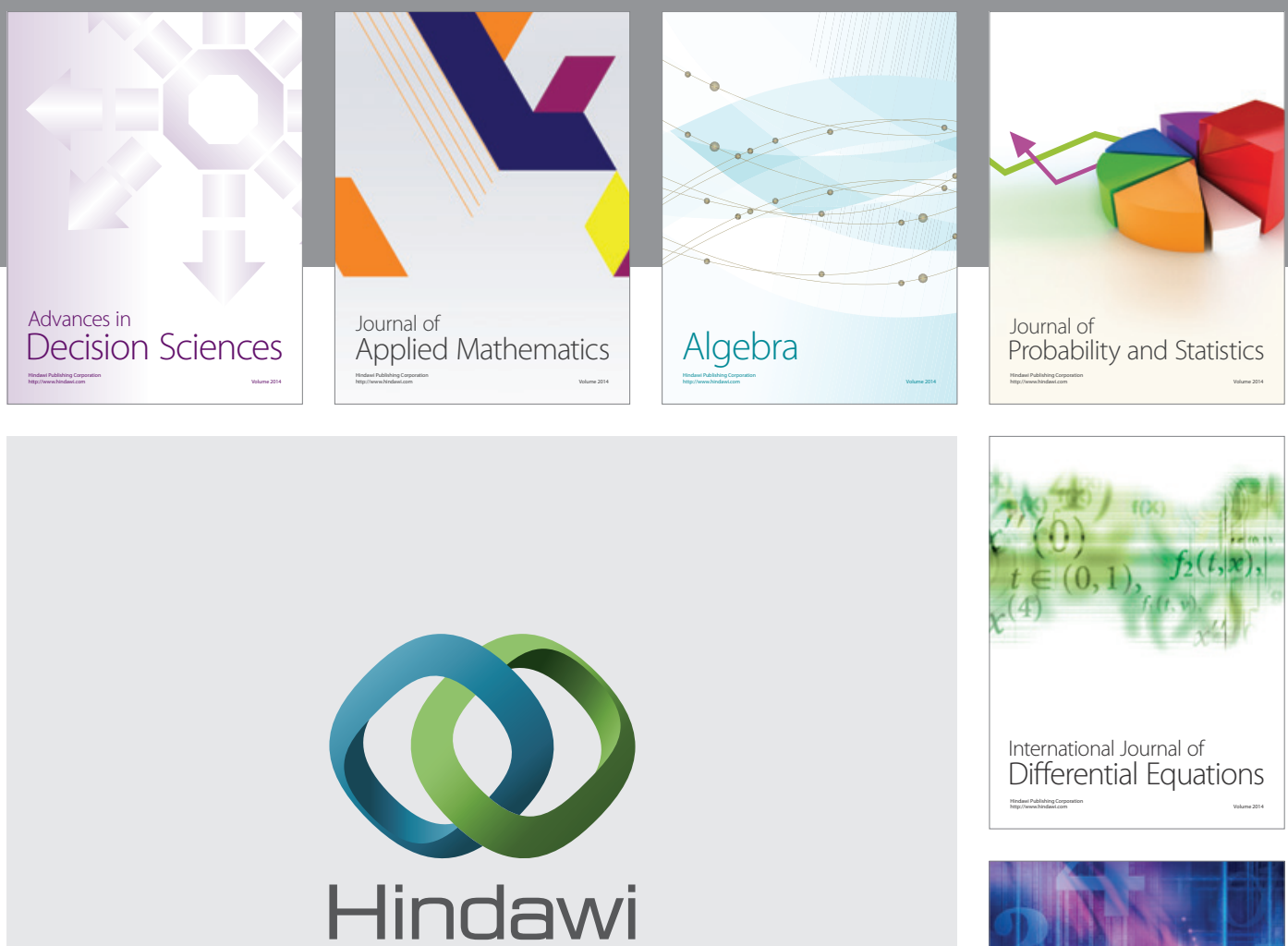

Submit your manuscripts at http://www.hindawi.com
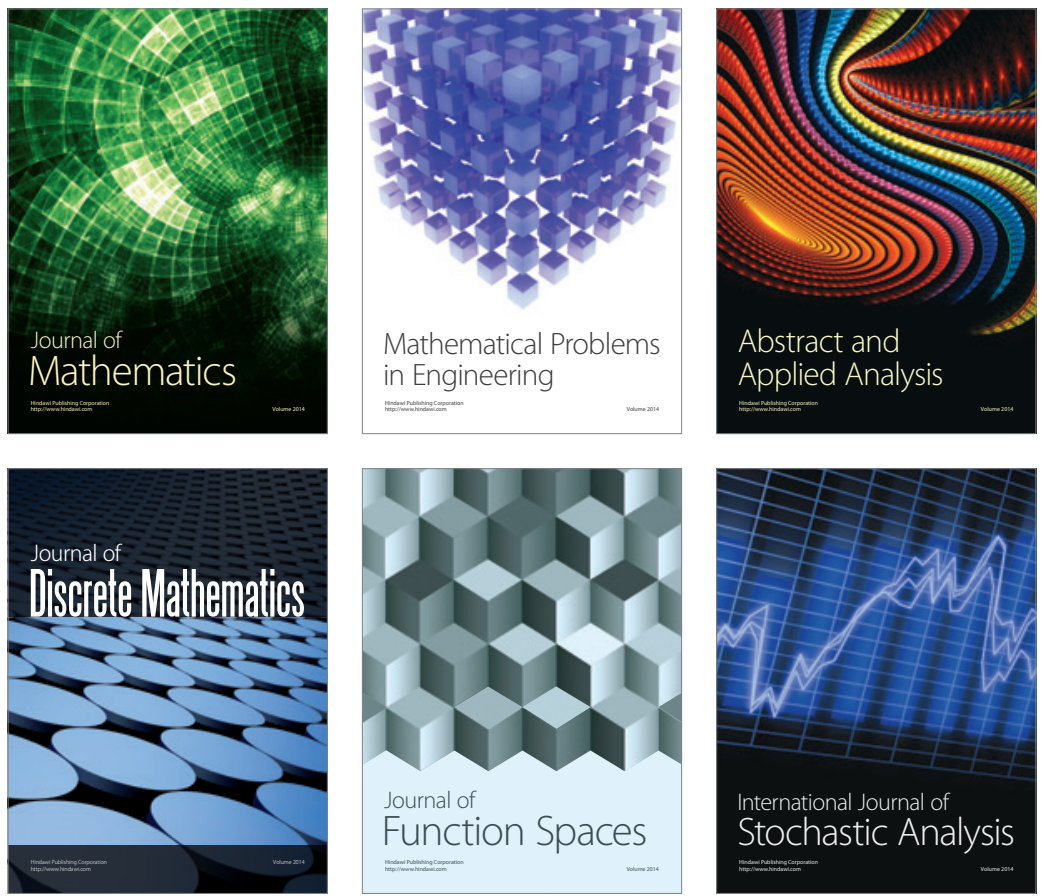

Journal of

Function Spaces

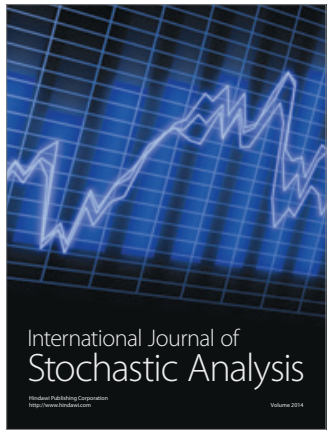

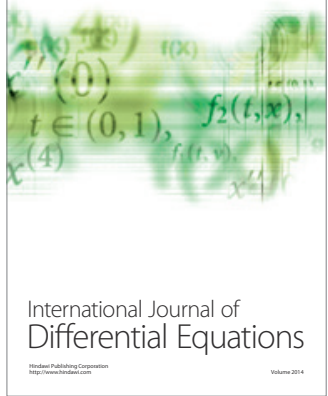
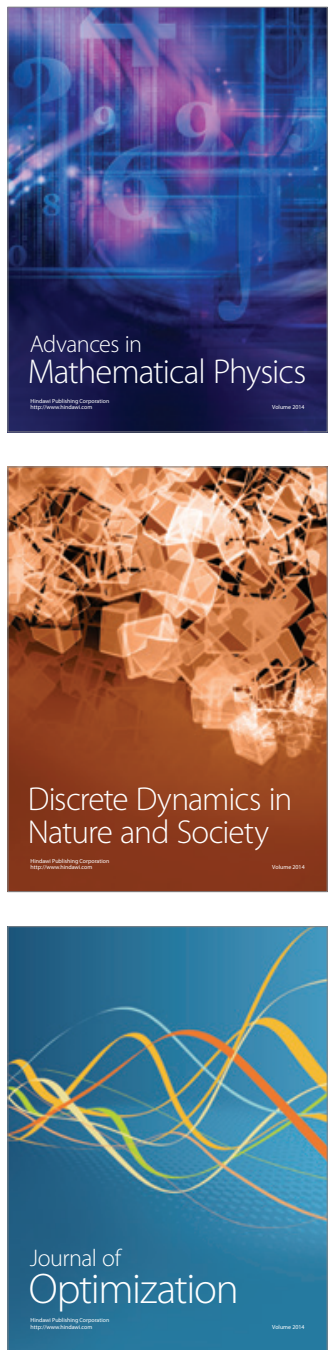